\title{
Can sub-optical-cycle parametric waveform synthesizers be seeded by separate bulk white-light supercontinua?
}

\author{
Roland E. Mainz, ${ }^{1,2}$ Giulio Maria Rossi, ${ }^{1,2}$ Giovanni Cirmi, ${ }^{1,2}$ Yudong Yang, ${ }^{1,2}$ Shih-Hsuan Chia, ${ }^{1,2}$ \\ Eiji J. Takahashi, ${ }^{3}$ Oliver D. Mücke, ${ }^{1,2}$ and Franz X. Kärtner ${ }^{1,2}$ \\ ${ }^{1}$ Center for Free-Electron Laser Science, Deutsches Elektronen-Synchrotron DESY, Notkestraße 85, 22607 Hamburg, Germany \\ ${ }^{2}$ Physics Department and The Hamburg Center for Ultrafast Imaging, University of Hamburg, \\ Luruper Chaussee 149, 22761 Hamburg, Germany \\ ${ }^{3}$ Extreme Photonics Research Group, RIKEN Center for Advanced Photonics, 2-1 Hirosawa, Wako, Saitama 351-0198, Japan \\ roland.mainz@cfel.de,franz.kaertner@cfel.de
}

\begin{abstract}
We present longitudinal-phase stability measurements between separate supercontinua generated in different bulk materials. The remarkably low relative phase jitter of few attoseconds allows multiple-white-light seeding schemes for flexible and ultrabroadband optical parametric waveform synthesizers.

OCIS codes: (320.7110) Ultrafast nonlinear optics; (190.4970) Parametric oscillators and amplifiers.
\end{abstract}

The current quest for optical parametric waveform synthesizers emitting intense electric-field transients $E(t)$ customsculpted on a sub-optical-cycle time scale is fuelled by numerous intriguing applications in strong-field physics and attoscience [1]. Such OP(CP)A-based synthesizers [2,3] are often seeded by an ultrabroad white-light supercontinuum (WL) generated in bulk materials. Although seeding of multi-channel parametric waveform synthesizers by a single, multi-octave WL (ideally passively CEP-stabilized [4]) is possible and already demonstrated [2], such single-WL seeding schemes impose extreme challenges on the synthesizer design: (i) the generation of a WL filament stable over its full bandwidth is far from trivial and, most critically, (ii) precise dispersion control, that permits final recompression to sub-cycle pulse durations $(<2 \mathrm{fs})$, requires very complex dispersion management schemes [2]. Implementing separate WLs, potentially even driven at different wavelengths, and created in different bulk materials, would thus add new degrees of freedom in the dispersion optimization and allow for unprecedented many-octave spectral coverage of such synthesizers. The immediate question arises: can sub-optical-cycle parametric waveform synthesizers in principle be seeded by separate bulk white-light supercontinua at all?

Since its first observation by Alfano and Shapiro in 1970 [5], WL generated by ultrashort laser pulses in bulk materials has attracted scientists' attention due to the created ultrabroadband spectra [6]. Importantly, the pioneering experiment of Bellini and Hänsch demonstrating phase coherence between two WL continua with 'negligible random phase jitter' also paved the way for optical frequency metrology based on femtosecond frequency combs [7]. WL generation via filamentation in bulk materials results from very complex highly nonlinear propagation [8], leading to its sensitive dependence on a number of parameters, both of the material and of the laser pulse used. Recently, also the influence of higher-order Kerr effect (HOKE) [9] has controversially been discussed. Therefore, the precise predictive power of numerical investigations concerning fine details of the spectral phase, CEP jitter and timing jitter [10] (resulting from amplitude-to-phase noise conversion) is at present unclear.
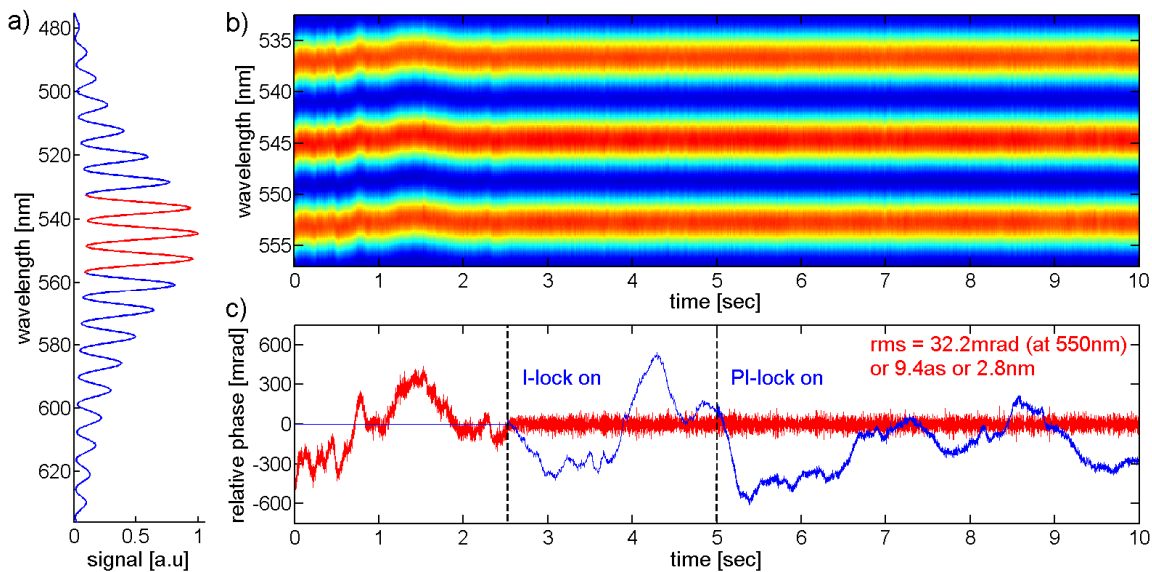

Fig. 1. (a) Single-shot spectral interference in the visible range. (b) $10 \mathrm{k}$ points sample showing the evolution of the spectral fringes over 10 seconds. (c) The phase of the fringes of each spectrum is plotted as red curve. Initially the phase is freely drifting mainly due to air and temperature fluctuations. After $2.5 \mathrm{~s}$ the slow I component of the feedback is activated (blue curve) and the phase is locked. After $5 \mathrm{~s}$ also the $\mathrm{P}$ component is switched on and the phase fluctuations shrink down even more reaching $32.2 \mathrm{mrad} \mathrm{rms}$ at $550 \mathrm{~nm}$ calculated over 1000 shots.

In this study, we experimentally determine a lower limit for the temporal coherence of WLs generated in separate few-mm-long bulk materials using 150-fs high-energy laser pulses from a cryogenic Ti:sapphire chirped-pulse amplifier. Note, single-WL seeding of synthesizers has the obvious advantage that the different spectral regions of 
the final pulse are originating from a common seed, so that, neglecting disturbances such as acoustic noise and temperature fluctuations/drifts, they are inherently phase-locked together. We believe this is also the only practical seeding scheme in sequential synthesizers [1]. Here the goal is to seed synthesizers with multiple WLs, each using the optimum material and thickness, with ultralow longitudinal phase jitter as required for sub-cycle waveform synthesis. Again, seeding with multiple WL becomes even more interesting, when pumping different supercontinua with different harmonics of the laser that are inherently phase locked.

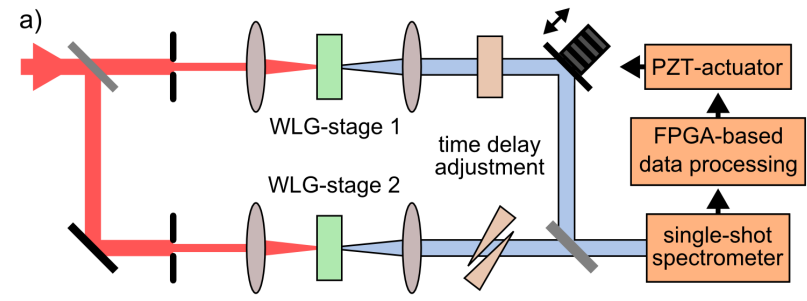

Fig. 2. (a) MZ-interferometer with two WL-generation stages. The spectrometer retrieves the phase of the fringe pattern and transmits it to the PI-loop to calculate the feedback. A fast PZT moves one of the mirrors in the interferometer, enabling the phase locking. (b) In blue color: spectrum of a 1.06- $\mu \mathrm{m}$-driven WL. In red colors: WL spectrum driven at $2.12 \mu \mathrm{m}$. The combined spectra extend over three octaves.

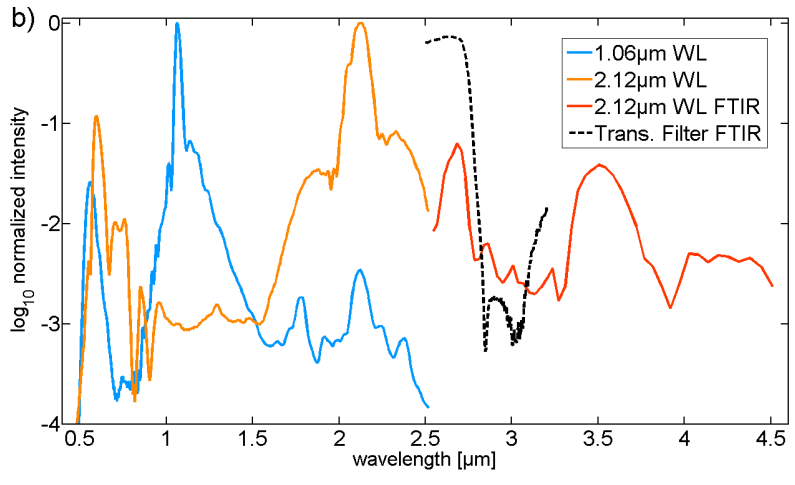

In the experiments, we implemented a Mach-Zehnder (MZ) interferometer, where the input pump pulse is split into two similar replicas that drive an independent WL in each of the two arms (see Fig. 2a). The materials employed in the WL generation are YAG and sapphire, of various thicknesses, while other materials are currently under investigation. The two WLs are then overlapped in space and time and their spectral interference is recorded. For this purpose, we built a custom spectrometer capable of single-shot and every-shot detection at the full $1 \mathrm{kHz}$ repetition rate. This is a crucial tool, since no conclusive statements on the temporal coherence can be made using only multi-shot integrated data. In addition, the spectrometer features an FPGA-based high-speed data evaluation system to retrieve the phase (via FFT) of the spectral fringes within $<300 \mu \mathrm{s}$. This fast processing speed allows to feed the phase back to a piezo-electric actuator inside the interferometer and to lock it with high precision over the full Nyquist bandwidth (DC to $500 \mathrm{~Hz}$ ).

\begin{tabular}{|l|l|c|c|}
\hline \multicolumn{1}{|c|}{ material 1 } & \multicolumn{1}{|c|}{ material 2 } & phase jitter $(\mathrm{rms})$ at $550 \mathrm{~nm}$ & timing jitter (rms) \\
\hline 2-mm YAG & 2-mm YAG & $32.2 \mathrm{mrad}$ & $9.4 \mathrm{as}$ \\
\hline 3-mm sapphire & 3-mm sapphire & $41.6 \mathrm{mrad}$ & 12.2 as \\
\hline 3-mm sapphire & 2-mm YAG & $51.2 \mathrm{mrad}$ & 15.0 as \\
\hline 1-mm sapphire & 3-mm sapphire & $64.4 \mathrm{mrad}$ & 18.8 as \\
\hline
\end{tabular}

Tab. 1. Dependence of the relative phase jitter on material and thickness of the two WL generation stages. The rms is calculated over 1000 single-shot samples.

The residual phase jitter after locking is extremely low: The two supercontinua exhibit single-shot relative phase stability of few tens of mrad rms consistent over an extended period of time (1 million shots or 16 minutes). Such phase noise corresponds to a few attoseconds time delay jitter (see Tab. 1). For comparison, integration over 4 laser shots yields $11.4 \mathrm{mrad} \mathrm{rms}, 10$ shots gives $5.4 \mathrm{mrad}$, and 100 shots $1.2 \mathrm{mrad}$. This residual noise includes mechanical vibrations of the Mach-Zehnder interferometer, phase-detection noise from the spectrometer $(<0.5 \mathrm{mrad})$ and nonlinear effects such as non-common-mode amplitude-to-phase coupling.

Encouraged by these results, we are now exploring more advanced schemes, in which a $2.12 \mu \mathrm{m}$ passively CEP stabilized pulse is used as pump of one WL, while its second harmonic $(1.06 \mu \mathrm{m})$ is employed to pump the second supercontinuum. Such an approach would allow seeding more efficiently an even broader bandwidth extending over three octaves (see Fig. 2b).

The presented data show that the intrinsic relative phase noise in the WL generation can be extremely low, practically negligible for sub-cycle waveform synthesis, since most of the remaining phase jitter in our experiment can most likely be attributed to acoustic noise, thermal fluctuations and laser instabilities.

\section{References:}

[1] C. Manzoni et al., Laser Photon. Rev. 9, 129 (2015).

[2] O. D. Mücke et al., IEEE J. Sel. Top. Quantum Electron. 21, 8700712 (2015); S.-H. Chia et al., Optica 1, 315 (2014).

[3] E. J. Takahashi et al., Nat. Commun. 4:2691 (2013).

[4] G. Cerullo et al., Laser \& Photonics Rev. 5, 323 (2011).

[5] R. R. Alfano and S. L. Shapiro, Phys. Rev. Lett. 24, 592 (1970).

[6] F. Silva et al., Nat. Commun. 3:807 (2012); M. Hemmer et al., Opt. Express 21, 28095 (2013); H. Liang et al., Opt. Lett. 40, 1069 (2015).

[7] M. Bellini and T. W. Hänsch, Opt. Lett. 25, 1049 (2000); T. W. Hänsch, Nobel Lecture, Rev. Mod. Phys. 78, 1297 (2006).

[8] A. Couairon and A. Mysyrowicz, Physics Reports 441, 47 (2007); L. Bergé et al., Rep. Prog. Phys. 70, 1633 (2007).

[9] B. Borchers et al., Opt Lett. 37, 1541 (2012).

[10] C. Manzoni et al., Phys. Rev. A 79, 033818 (2009). 